\title{
Heterodyne displacement interferometer, insensitive for input polarization
}

\author{
Arjan J. H. Meskers, ${ }^{*}$ Jo W. Spronck, and Robert H. Munnig Schmidt \\ Delft University of Technology, Department of Precision and Microsystems Engineering, Mekelweg 2, 2628 CD Delft, The Netherlands \\ *Corresponding author: a.j.h.meskers@tudelft.nl
}

Received February 3, 2014; revised February 21, 2014; accepted February 21, 2014;

posted February 24, 2014 (Doc. ID 205914); published March 24, 2014

\begin{abstract}
Periodic nonlinearity (PNL) in displacement interferometers is a systematic error source that limits measurement accuracy. The PNL of coaxial heterodyne interferometers is highly influenced by the polarization state and orientation of the source frequencies. In this Letter, we investigate this error source and discuss two interferometer designs, designed at TU Delft, that showed very low levels of PNL when subjected to any polarization state and/or polarization orientation. In the experiments, quarter-wave plates (qwps) and half-wave plates (hwps) were used to manipulate the polarization state and polarization orientation, respectively. Results from a commercial coaxial system showed first-order PNL exceeding $10 \mathrm{~nm}$ (together with higher order PNL) when the system ceased operation at around $\pm 15^{\circ}$ hwp rotation or $\pm 20^{\circ}$ qwp rotation. The two "Delft interferometers," however, continued operation beyond these maxima and obtained first-order PNLs in the order of several picometers, without showing higher order PNLs. The major advantage of these interferometers, beside their high linearity, is that they can be fully fiber coupled and thus allow for a modular system buildup. (®) 2014 Optical Society of America

OCIS codes: (120.0120) Instrumentation, measurement, and metrology; (120.3180) Interferometry; (120.3940)

Metrology; (120.4570) Optical design of instruments; (120.4820) Optical systems; (120.5050) Phase measurement.

http://dx.doi.org/10.1364/OL.39.001949
\end{abstract}

Laser interferometry is an often applied measurement method in several fields of research, since it allows for noncontact measurements. It is especially preferred in the field of metrology, because of its direct traceability to the length standard [1]. Interferometry is also used in lithography machines in the semiconductor industry, gravitational wave detection [2], coordinate measuring machines, and as a calibration tool for other measurement devices, such as capacitive sensors, inductive sensors, and optical encoders.

Many types of displacement interferometer systems can be distinguished; this publication deals with heterodyne displacement interferometry using a stabilized He-Ne laser ( $\lambda=632.8 \mathrm{~nm})$ combined with two acoustooptic modulators for generating two (fixed-offset) source frequencies.

Industrial manufacturing processes currently operate with measurement errors at the subnanometer level and will require even smaller errors in the near future [3]. When operating at this level, a heterodyne interferometer system is hampered by many error sources. The main error sources are the frequency stability of the laser source, noncommon optical pathway variations due to variations in the refractive indices of optical transport media, system alignment (i.e., cosine error, Abbé error), optical wavefront quality combined with beam walkoff [4]], and periodic nonlinearity (PNL) in the measured phase [5-10]. Operating in vacuum will improve the obtained measurement error. However, PNL in the measurand remains a substantial error source.

PNL manifests itself primarily in traditional heterodyne systems with coaxial beams. Such beams contain two linearly polarized frequencies that are orthogonally oriented. These frequencies may mix (due to "frequency leakage"), resulting in periodic errors that are superimposed on the obtained displacement data. This type of error repeats itself each fraction of a wavelength-hence "periodic"-and is caused by imperfect polarization orthogonality and linearity of the source frequencies combined with imperfect polarizing optics and nonideal alignment [5] .

There are several approaches for reducing PNL in coaxial interferometer systems. Physically reducing PNL is one of the most costly methods, since it involves expensive high-quality optics and routine individual system alignment. This reduction approach is based on the quality of the coaxial beam's source frequency orthogonality together with the quality of the linear polarizations. If these are of high quality, it is also essential that highquality polarized optics are applied for beam splitting, combined with alignment [ $\underline{5}-\underline{9}]$.

A less expensive approach is to digitally compensate for the PNL through analytical modeling $[5,9-11]$. The advantage of this method is that it typically ensures PNL below $1 \mathrm{~nm}$ for small system imperfections, without changing the interferometer configuration. Figure $\underline{1}$
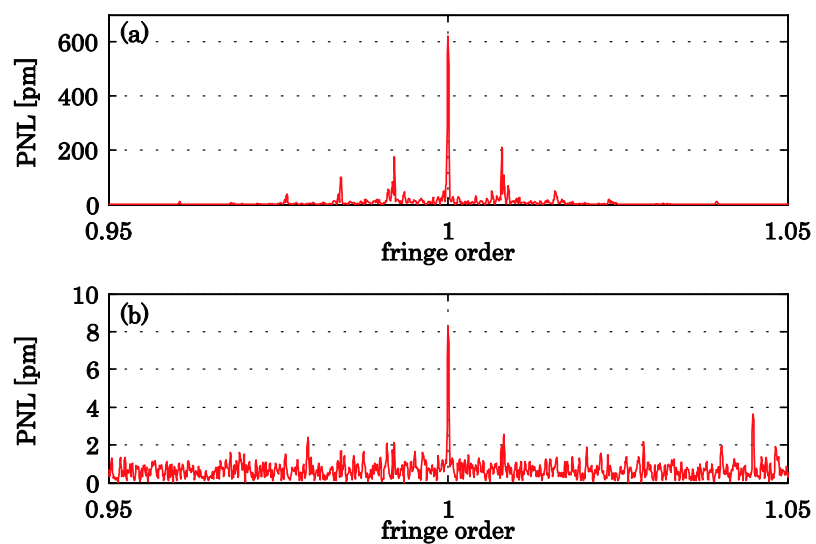

Fig. 1. Demonstration of digital PNL compensation in open air, using an Agilent interferometer system [21]. The results show the amplitudes of first-order PNL over "fringe order" [Eq. (1)], calculated by an FFT performed on displacement data of a mirror mounted on an Aerotech stage (ABL10100LT), displacing at a constant velocity of $0.5 \mathrm{~mm} / \mathrm{s}$. Compensation is (a) $\mathrm{OFF}$ and (b) ON, demonstrating a PNL reduction factor of $\sim 75$. 
shows as an example the capabilities of digital PNL compensation. However, some of these methods require (periodic) calibration, a minimum displacement per timeframe, and additional calculation time compared to uncompensated systems. These drawbacks make PNL compensation algorithms difficult or unsuitable to apply to (quasi) static or small stroke and near-real-time operating systems, depending on the applied algorithm.

The ideal solution would be to not have PNL in the first place, so that compensation is not needed. This can be achieved by keeping the two source frequencies spatially separated throughout the interferometer system until detection takes place [12-19]. Spatial separation prevents frequency leakage occurring, thereby prohibiting PNL, but it also leads to possible optical path length inequality during source frequency delivery. The limitation of these interferometer configurations is their special and often complicated optical layouts [18] that limit their applicability.

The interferometer designs discussed in this Letter [15-17], designed at TU Delft, are of equal optical complexity compared to coaxial systems and use spatially separated source frequencies (generated using [20]). Furthermore, the Delft designs allow for a high degree of system integration, as fiber coupling is possible for both frequency delivery and signal detection. In this Letter we investigate the PNL sensitivity versus polarization state and polarization orientation of two Delft interferometers and compare the results with a traditional coaxial system [21] that was subjected to equal polarization manipulation.

As measurement targets, mirrors were mounted on a single degree of freedom (DoF) Aerotech stage, which was programmed to displace at constant velocity [see the setup in Fig. 2(a)]. PNL is visualized by fitting an $x$ th-order polynomial through the obtained displacement data. This fit is subsequently subtracted from the displacement data themselves, thereby removing macroscale motion (e.g., air turbulence and mechanical vibrations) and quasi-static effects (i.e., thermal deformation or source frequency drift). The resulting dataset is then fast Fourier transformed, revealing the location (frequency, i.e., fringe order) and amplitude of the PNL [22-24]. During the fast Fourier transform (FFT) analysis, a "flat top" window has been applied, providing the best amplitude accuracy for determination of the PNL and noise level amplitudes. Depending on the polarization orientation (i.e., misalignment) and state (i.e., quality), first-order or higher PNL will show up in the FFT data. The frequency locations of the PNL order are calculated using

$$
f_{\mathrm{PNL}}=\left(k \cdot N \cdot v_{\text {target }}\right) / \lambda,
$$

where $k$ is a positive integer value expressing the $x$ thorder PNL, $N$ represents the number of times that the beam traverses between the interferometer and the measurement target, $v_{\text {target }}$ is the target velocity (i.e., the Aerotech stage), $\lambda$ is the wavelength used $(632.8 \mathrm{~nm})$, and $f_{\mathrm{PNL}}$ is the PNL frequency in the FFT spectrum. Knowing these frequencies helps during the identification process by showing peaks at integer intervals, as in Fig. 1. Processing the data as described, combined with $f_{\mathrm{PNL}}$ in the kilohertz range, makes the measurement method insensitive for long time constant effects, which helps in lowering the noise level.

The FFT analysis used for visualization of PNL also includes peaks caused by mechanical vibrations picked up during displacement measurements. It is thus important to know whether a PNL peak coincides with stage vibrations or not; otherwise false PNL results are obtained. Therefore, target motion was investigated with a capacitive probe [25], which has a low noise level and is inherently free of PNL. Measurements were performed at different stage locations (due to the capacitive probe's short measurement stroke, $100 \mu \mathrm{m}$ ), after which the obtained data were processed in the same way as previously described. Analysis showed no vibrations at the frequencies where PNL manifests (i.e., fringe order locations).

The following paragraphs will discuss the outcomes of measurements performed in the lab. In the first measurement, the two linear perpendicular polarizations of the coaxial beam of the commercial system [21] were rotated (i.e., misalignment) with respect to the interferometer optics. This was done using a half-wave plate (hwp) at wpl [see Fig. 2(a)]. Figure 3(a) shows the results when the hwp rotated the polarizations between about $\pm 15^{\circ}$ clockwise and counterclockwise. At these maxima, operation ceased, because the PNL became too large for the electronics to continue measuring. Noticeable is the $\mathrm{V}$ shape of the first PNL order and the bowl shape of the
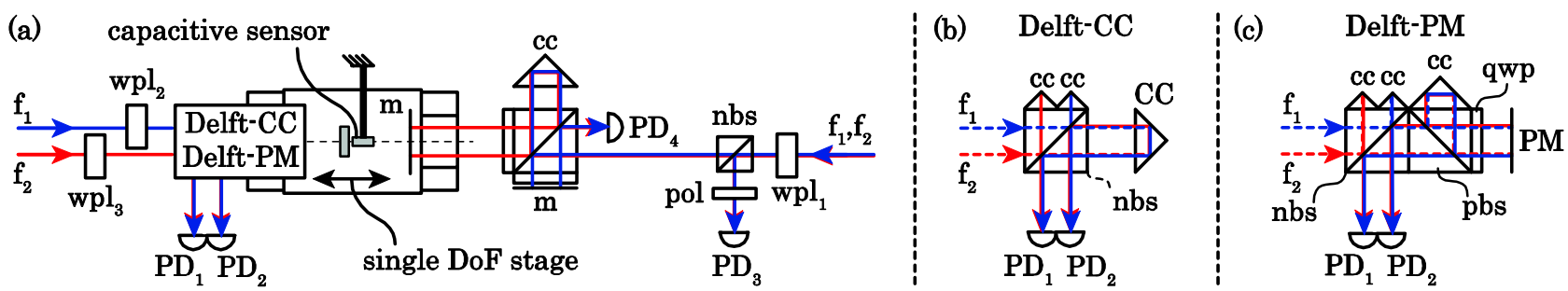

Fig. 2. (a) Experimental setup overview; two interferometers measure target displacement simultaneously. A capacitive probe [25] is used for vibration analysis of the stage's motion. On the left either the Delft-CC or Delft-PM interferometer is included (together with [20]), while on the right one finds a commercial (coaxial) interferometer system [21]. The optical pathways of the (b) Delft-CC and (c) Delft-PM interferometers are shown. $f_{x}$, source frequency; $\mathrm{PD}_{x}$, photodetector; pol, polarizer; nbs, neutral beam splitter; pbs, polarized beam splitter; qwp, quarter wave plate; cc, corner cube mirror; CC, corner cube target mirror; PM, plane target mirror; $\mathrm{wpl}_{x}$, waveplate. Note that the two Delft interferometers consist of separate optical components, whereas the commercial interferometer [21] is an optical monolith. 

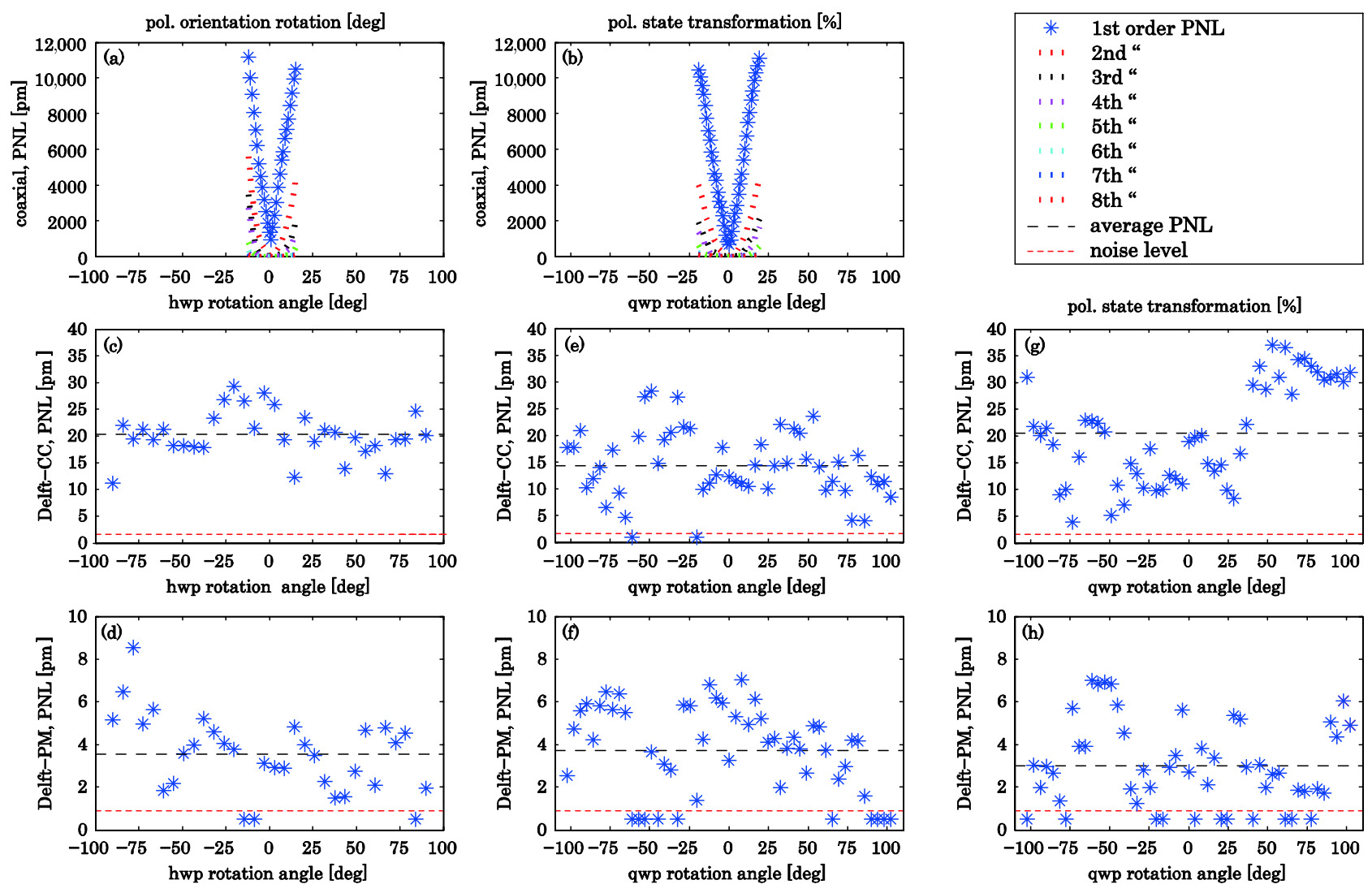

Fig. 3. Measurement results showing PNL amplitudes of three interferometers. (a) Both source frequencies were rotated (coaxial beam) using a hwp; (c), (d) only $f_{1}$ was rotated. (b) For both source frequencies, their linear polarization states were transformed from linearly polarized toward left and right circularly polarized; (e), (f) this was only done for $f_{1}$ (i.e., $f_{2}$ remained linear). (g), (h) The polarization state of $f_{1}$ was manipulated again from linearly polarized to left- and right-circularly polarized, while $f_{2}$ was kept continuously circularly polarized. Note that below noise level indicates that no PNL was observed.

second- and higher-order PNLs, of which the shape flattens as the PNL order increases [22,23].

Two Delft interferometers (Delft-C $\bar{C}$ with an $\lambda / 4$ optical resolution [Fig. 2(b)] and Delft-PM with an $\lambda / 8$ optical resolution [17] [Fig. 2(c)]) were tested in an equal manner, but with two source beams rather than a single (coaxial) beam. For these systems, a hwp was inserted into beam $f_{1}$ [wpl $\mathrm{w}_{2}$, Fig. 2(a)] while $f_{2}$ was kept linearly (vertically) polarized; the results are shown in Figs. 3(c) and 3(d). Neither of the Delft concepts showed a significant change in operational performance during polarization rotation of $f_{1}$. Both interferometers continued operation even at $90^{\circ}$ relative rotation, where $f_{1}$ 's polarization is perpendicular to $f_{2}$ 's polarization (indicating that $f_{1}$ and/or $f_{2}$ was not $100 \%$ linearly polarized). Both Delft interferometers showed for all measurements only first-order PNL without higher orders. The Delft-CC system obtained an average first-order PNL of $\sim 20 \mathrm{pm}$, while the Delft-PM system obtained an average below $4 \mathrm{pm}$. This verifies that these interferometers are insensitive to the polarization orientations of their source frequencies.

The low levels of PNL depicted in Figs. 3(c)-3(h), are attributed to secondary effects, such as ghost reflections [26] caused by nonantireflection-coated optical components. These reflections also caused a (steady) halffringe-order PNL amplitude of about $\sim 170 \mathrm{pm}$, which appeared to be directly related to system alignment.
Antireflection-coated monolithic optics solve this halffringe-order PNL, as the system in [21] proves.

The sensitivity of the commercial system [21] regarding polarization state versus PNL was tested using a quarter-wave plate (qwp) at wpl $_{1}$ (see Fig. 2). Figure 3(b) shows the results of the polarization transformation of the coaxial beam, where rotation of the qwp transforms the polarization state from linear into left and right circular. At about $\pm 20^{\circ}$ qwp rotation, the system again ceased operation, for the same reason as in the hwp rotation test.

For each Delft interferometer, two tests were performed using qwps for obtaining the PNL sensitivity versus polarization state. The first test involved insertion of a qwp in $f_{1}\left(\mathrm{wpl}_{2}\right.$, Fig. 2) while keeping $f_{2}$ linearly (vertically) polarized. The results in Figs. 3(e) and 3(f) again show no significant impact on PNL. The PNL magnitude for the Delft-CC improved to an average first-order PNL of $\sim 15 \mathrm{pm}$, while the Delft-PM design obtained a firstorder PNL average below $4 \mathrm{pm}$.

The second qwp test with the Delft interferometers involved insertion of one qwp into $f_{1}$ and one into $f_{2}$ [at $\mathrm{wpl}_{2}$ and $\mathrm{wpl}_{3}$, respectively (Fig. 2)]. The qwp in $f_{1}$ manipulated the polarization state again from linear toward full left- and right-circularly polarized, while the qwp at $\mathrm{wpl}_{3}$ kept $f_{2}$ fully circularly polarized. This resulted in an average first-order PNL of $\sim 20 \mathrm{pm}$ for the Delft-CC design and an average first-order PNL of 
$\sim 3 \mathrm{pm}$ for the Delft-PM design. This verifies that these interferometers are insensitive to the polarization states of their source frequencies.

The repeatability of the measurements for the Delft interferometers, as shown in Fig. 3, was tested by repeated measurements at the same wave plate angle. The average repeatability was about $\pm 4 \mathrm{pm}$ for the Delft-CC and \pm 2 pm for the Delft-PM system.

In this Letter we experimentally determined the amplitudes of the PNLs of three heterodyne interferometers by subjecting them to polarization-manipulated source frequencies. This manipulation involved wave plates that rotated the heterodyne frequencies' polarization orientation or transformed their polarization state. The tested commercial system [21] showed first-order (and up to eighth-order) PNL with amplitudes exceeding $10 \mathrm{~nm}$ when exposed to approximately $\pm 15^{\circ}$ hwp rotation or $\pm 20^{\circ}$ qwp rotation; these were the maxima at which measuring stopped because the PNL became too large for the electronics to continue measuring. The two Delft interferometers, on the other hand, continued operation regardless of any polarization manipulation and obtained average first-order PNLs of $20 \mathrm{pm}$ (Delft-CC) and $4 \mathrm{pm}$ (Delft-PM). The results indicated no significant relation between PNL and polarization imperfections. The Delft interferometers are thus insensitive to any imperfection in source frequency polarization, while coaxial systems show high sensitivity.

This research has demonstrated that more accurate displacement measuring interferometers can be achieved using the discussed interferometers (i.e., increased linearity compared to current state-of-the-art systems [21]). Moreover, the discussed interferometers can be fully fiber coupled, leading to modular systems that are easier to implement in a host system compared to current coaxial systems that are bound to free-space beam delivery due to the optical layout of the interferometer optics.

This work was supported by the Dutch IOP (IPT04001) in The Netherlands. The authors are thankful for the support by Agilent Technologies, who provided equipment used during this research.

\section{References and Notes}

1. Th. Udem, R. Holzwarth, and T. W. Hansch, Nature 416, 233 (2002).

2. G. M. Harry, Class. Quantum Grav. 27, 084006 (2010).

3. C. Wagner and N. Harned, Nat. Photonics 4, 24 (2010).

4. A. J. H. Meskers, D. Voigt, and J. W. Spronck, Opt. Express 21, 17920 (2013).

5. S. J. A. G. Cosijns, H. Haitjema, and P. H. J. Schellekens, Precis. Eng. 26, 448 (2002).

6. G. Fedotova, Meas. Tech. 23, 577 (1980).

7. R. Quenelle, Hewlett Packard J. 34, 10 (1983).

8. C. Sutton, J. Phys. E 20, 1290 (1987).

9. W. Hou and G. Wilkening, Precis. Eng. 14, 91 (1992).

10. C. Wu and R. D. Deslattes, Appl. Opt. 37, 6696 (1998).

11. W. Hou, Precis. Eng. 30, 337 (2006).

12. M. Tanaka, T. Yamagami, and K. Nakayama, IEEE Trans. Instrum. Meas. 38, 552 (1989).

13. C.-M. Wu and R. D. Deslattes, Appl. Opt. 38, 4089 (1999).

14. T. Schmitz and J. Beckwith, J. Mod. Opt. 49, 2105 (2002).

15. K.-N. Joo, J. D. Ellis, J. W. Spronck, P. J. M. van Kan, and R. H. M. Schmidt, Opt. Lett. 34, 386 (2009).

16. K.-N. Joo, J. D. Ellis, E. S. Buice, J. W. Spronck, and R. H. M. Schmidt, Opt. Express 18, 1159 (2010).

17. J. D. Ellis, A. J. H. Meskers, J. W. Spronck, and R. H. M. Schmidt, Opt. Lett. 36, 3584 (2011).

18. C. Weichert, P. Kochert, R. Koning, J. Flugge, B. Andreas, U. Kuetgens, and A. Yacoot, Meas. Sci. Technol. 23, 094005 (2012).

19. J. Lawall and E. Kessler, Rev. Sci. Instrum. 71, 2669 (2000).

20. Spatial heterodyne frequency generation Delft system: Thorlabs stabilized He-Ne laser HRS015, ISOMET acousto-optic modulators OAM 1141-T40-2 and drivers 531C-L (39 and $41 \mathrm{MHz}$ ), and phase measurement readout according to Agilent Technologies phase measurement board N1225A.

21. Agilent Technologies interferometer E1826G (optical resolution of 4), Zeeman laser source $5517 \mathrm{D}$, and phase measurement board N1225A.

22. V. G. Badami and S. R. Paterson, Precis. Eng. 24, 41 (2000).

23. T. L. Schmitz, D. Chu, and L. Houck III, Meas. Sci. Technol. 17, 3195 (2006).

24. C. Schluchter, V. Ganguly, D. Chu, and T. L. Schmitz, Precis. Eng. 35, 241 (2011).

25. Capacitive probe 2805MSE A9089 and electronic readout using MicroSense, LLC, model 4810.

26. C.-M. Wu, Opt. Commun. 215, 17 (2003). 